\title{
Ultrafine Hydrogel Nanoparticles: Synthetic Approach and Therapeutic Application in Living Cells**
}

\author{
De Gao, Hao Xu, Martin A. Philbert, and Raoul Kopelman*
}

The entry of drug payloads into the circulatory system is met by rapid removal by resident macrophages. The almost immediate drop in circulating levels of the therapeutic agent has been identified as a major obstacle for efficient drug delivery. ${ }^{[1]}$ Various physical properties determine the fate of NP-based (NP = nanoparticle) drug-delivery platforms in vivo. Size, surface charge, and surface hydrophobicity of the particles all affect the ability of macrophages to remove them from circulation and, hence, their bioavailability. Large particles ( $>200 \mathrm{~nm}$ diameter) are more easily captured by macrophages. Ultrafine nanoparticles can avoid resident macrophage capture and are thus more likely to be eventually removed from the body by renal clearance, thereby decreasing the potential for bioaccumulation. The use of ultrafine materials of appropriate size and composition for the delivery of potent and effective therapeutics may mitigate some of the problems associated with drug delivery to the central nervous system (CNS) as well as management of other diseases.

Ultrafine polyacrylamide-based hydrogels are an example of the many interesting materials used for drug delivery in vivo. The neutral surface properties of the hydrophilic polyacrylamide-based nanoplatforms reduce uptake by macrophage and make them a candidate for development as a useful therapeutic entity. ${ }^{[2]}$ Lower protein adsorption and high water content further decrease the opsonization by plasma proteins in the bloodstream and aid in the evasion of circulating and tissue-based macrophages. The size of polyacrylamide-based nanoparticles can be easily modulated by synthetic approaches. Furthermore, polyacrylamide can easily copolymerize with other monomers to introduce functional groups; thus, targeting tags can be incorporated to guide drug delivery. All this suggests that polyacrylamide-based hydrogels may be used as a novel class of safe nanosized drug carriers for the in vivo delivery of a variety of therapeutic moieties.

[*] Dr. D. Gao, Prof. R. Kopelman

Department of Chemistry

University of Michigan

Ann Arbor, MI 48109-1055 (USA)

Fax: (+1) 734-936-2778

E-mail: kopelman@umich.edu

Dr. H. Xu, Prof. M. A. Philbert

Department of Environmental Health Sciences

University of Michigan

Ann Arbor, MI 48109-2029 (USA)

[**] We thank Dr. Kei Sun for the help with TEM images. This work was supported by National Cancer Institute UIP contract N01-CO37123.

0 Supporting information for this article is available on the WWW under http://www.angewandte.org or from the author.
Photodynamic therapy (PDT), a promising new treatment for certain types of cancer, has received considerable attention in recent years. ${ }^{[3,4]}$ The principle of PDT is that optically excited photosensitizer molecules, localized in cancer tissues, transfer their energy to molecular oxygen to form highly reactive singlet oxygen $\left({ }^{1} \mathrm{O}_{2}\right)$, which kills living cells. The subcellular localization of photosensitizers depends on the photosensitizer's nature and the cell lines studied. Among the "second-generation" photosensitizers, meta-tetra(hydroxyphenyl)chlorin ( $m$ THPC) has recently received extensive attention because of its high phototoxicity at very low concentrations, or low light levels, and it has recently been approved in the European Union for head and neck cancer therapy. ${ }^{[4]}$ However, a specific formulation is required to deliver the hydrophobic drug. This is because the injectable $m$ THPC, for PDT clinical trials and for most in vivo studies reported in the literature, still uses the standard solution (ethanol/PEG400/water) formulation. In this way, after administration, the hydrophobic molecules are diluted in the biological environment. Eventually the $m$ THPC molecules precipitate out and stick onto cell or tissue surfaces, causing side effects. ${ }^{[5]}$ Although a few reports show the encapsulation of $m$ THPC into sub-200-nm silica or poly(lactic-co-glycolic acid) (PLGA) nanoparticles, ${ }^{[6]}$ size and surface issues, as well as application in a biological environment, remain open.

Herein, a novel synthetic approach is described for obtaining ultrafine hydrophilic polyacrylamide-based nanoparticles. Used as an example, $m$ THPC-encapsulating nanoparticles are synthesized. The behaviors of the drug molecule, within nanoparticles and in solution, are chemically compared for their phototoxicity. Finally, their effectiveness in killing living cells was evaluated.

It is well-known that for inverse microemulsions the droplet size is determined by the molar ratio of water to surfactant, $\left[\mathrm{W}_{0}\right] /[$ surfactant $] .^{[7]}$ Also, droplet stability during polymerization depends on the volume of the water droplets. The volume reduction of the water pool and the increase in surfactant, in the inverse microemulsion, makes the droplet more rigid and thus decreases the possibility of droplet collisions during polymerization. Thus, small-size nanoparticles can be obtained. Experiments have shown that by changing the molar ratio of water to surfactants one could control both size and shape of the nanoparticles. ${ }^{[7]} \mathrm{We}$ chose to emulsify both the monomers and cross-linkers directly into hexane, without water, to get the smallest polyacrylamide nanoparticles. First, the ultrafine blank polyacrylamide particles were synthesized. Acrylamide (AAm) monomer and cross-linker $N, N$-methylenebis(acrylamide) (MBA) were suspended in a hexane solution containing surfactant $(\mathrm{AOT}=$ 
bis(2-ethylhexyl)sulfosuccinate sodium salt). The suspension was stirred and sonicated until the solid was emulsified into hexane. Then polymerization was initiated with ammonium persulfate (APS) and $N, N, N^{\prime}, N^{\prime}$-tetramethylethylenediamine (TMEDA) under argon. Nanoparticles less than $2 \mathrm{~nm}$ in diameter, with very narrow distributions, were observed by dynamic-light-scattering measurements with an ultrafineparticle analyzer (Nanotrac NPA250, Microtrac Inc, PA). High-resolution TEM measurements also agreed with the dynamic-light-scattering results. Comparison experiments showed that the common microemulsion (water/hexane/ AOT) system generated $20-\mathrm{nm}$ diameter polyacrylamide nanoparticles, with a relatively wider distribution, ${ }^{[8]}$ while much smaller nanoparticles, only 1-2 $\mathrm{nm}$ in diameter, with a very narrow distribution, were obtained by the new synthetic approach.

Because $m$ THPC is a hydrophobic component, it suffers from precipitation in biological environments. A specific formulation is required to obtain an injectable drug. This limits the potential therapeutic applications for $m$ THPC. We further experimentally examined whether ultrafine hydrophilic nanoparticles can encapsulate hydrophobic drug molecules into their small sizes to circumvent the problem. To encapsulate the hydrophobic molecules into the hydrophilic nanoparticles, we used a small volume of methanol as solvent to dissolve $m$ THPC, AAm, as well as MBA, and emulsify the polar solution into the nonpolar, AOT-containing hexane. Most of the methanol was evaporated to minimize the [methanol]/[AOT] ratio. Typically, $m$ THPC-encapsulating nanoparticles with a diameter of $2-3 \mathrm{~nm}$ were observed (Figure 1). The TEM images confirmed that our synthetic approach can indeed produce ultrafine dye-loading nanoparticles.

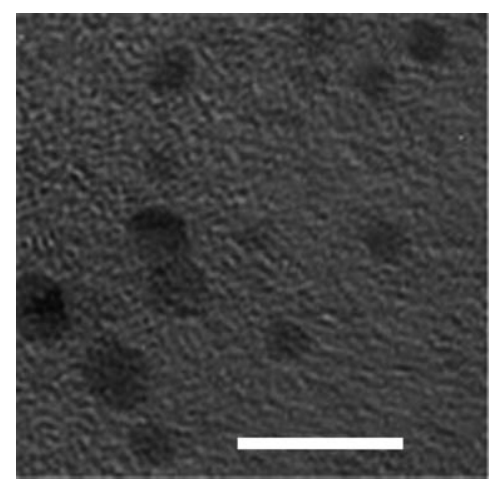

Figure 1. TEM image of $m$ THPC-encapsulating polyacrylamide nanoparticles. Scale bar: $10 \mathrm{~nm}$.

The molecular behaviors of drugs in nanoparticles will affect drug treatment efficiency, and it is especially interesting to obtain information on the drug molecule's behaviors in such small nanoparticles. The same concentration of free $m$ THPC in ethanol, in phosphate-buffered saline (PBS), and its nanoparticles in PBS was spectrophotometrically measured. Absorption and excitation spectra (excited at $710 \mathrm{~nm}$ ) were recorded for aggregation studies in these solutions. The absorption spectrum and the excitation spectrum of free $m$ THPC are identical in ethanol, which indicates that the $m$ THPC molecules did not aggregate in this solution. ${ }^{[9]}$

Also, our results showed that the absorption spectrum of $m$ THPC-containing nanoparticles in PBS did matches with their excitation spectrum. These experiments demonstrated that $m$ THPC is evenly distributed within the nanoparticles and it behaves like free $m$ THPC molecules in ethanol solution. However, for free $m$ THPC in PBS, pH 7.4 buffer, the comparison of absorption and excitation spectra showed that the hydrophobic dye did aggregate. This result indicates that the polyacrylamide nanoparticle design can protect the dye from aggregation by encapsulating it inside the nanomatrix.

We were also concerned with whether $m$ THPC would leach out from such small nanoparticles. Our experiments showed that the $m$ THPC-containing nanoparticle excitation spectra did not change after monitoring for two days. This result indicates that the dye is confined within the nanoparticles, and that no significant dye leaching occurred during the two-day period.

To evaluate the singlet oxygen production in aqueous solution, the singlet oxygen production efficiency was measured fluorometrically by using anthracenedipropionic acid (ADPA) disodium salt, for free $m$ THPC in ethanol and in PBS, as well as its nanoparticles in PBS. The concentration of ADPA was fit, linearly, to $[\mathrm{ADPA}]=1-0.0030 t$ and [ADPA] $=1-0.0011 t$, for free $m$ THPC in ethanol and PBS buffer, respectively, while for $m$ THPC-encapsulating nanoparticles in PBS, [ADPA] $=1-0.0027 t$ was obtained (where the coefficient in each case represents the slope of the fit (in $\min ^{-1}$ ) in Figure $2 b$ ). This result indicates that the singlet

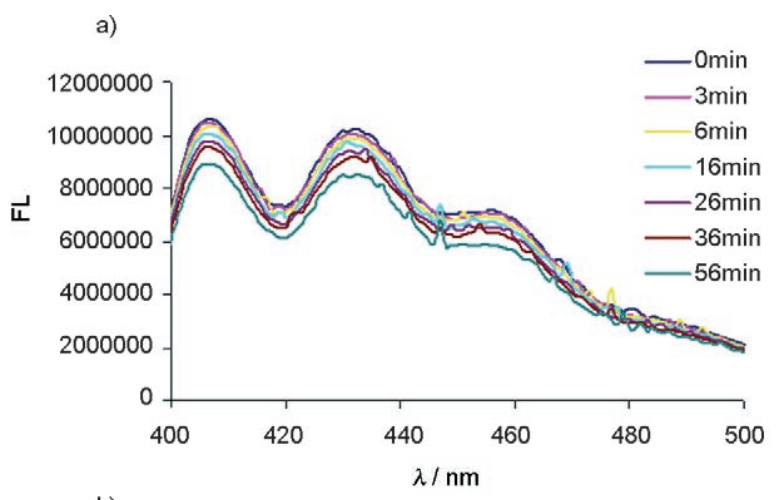

b)

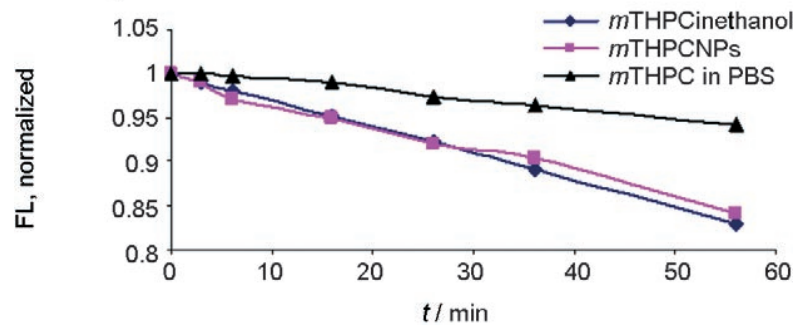

Figure 2. a) ADPA fluorescence (FL) in the presence of $0.3-\mu \mathrm{M} m \mathrm{mTHC}$ nanoparticles under laser illumination. b) Curves of ADPA fluorescence intensity versus time in the presence of free $m$ THPC $(0.3 \mu \mathrm{M})$ in ethanol and in PBS buffer, as well as the same concentration of $m T M P C$ in nanoparticles in PBS buffer. 
oxygen production efficiency of $m$ THPC had not deteriorated significantly as a result of encapsulation within the ultrafine hydrophilic polyacrylamide matrix. The photodynamic efficiency of $m$ THPC in nanoparticles (in buffer) is thus similar to that of free $m$ THPC molecules in ethanol. Although the singlet oxygen lifetime usually is short in aqueous environments, ${ }^{[4]}$ singlet oxygen can still effectively diffuse out of the ultrafine hydrogel and kill cells. This result further confirms the advantages of drug-containing ultrafine nanoparticles.

For the living cell study, the first thing studied was the $m$ THPC molecule distribution. Stock solutions of free $m$ THPC $\left(0.5 \mathrm{mg} \mathrm{mL}^{-1}\right)$ were made in polyethylene glycol, ethanol, and PBS buffer (3:2:5). Further dilutions were performed in PBS just before the experiment. After incubation with $m$ THPC for $1 \mathrm{~h}$ without light, cells were washed with Hanks buffer three times to remove free $m$ THPC. The cells in fresh medium were excited at $488 \mathrm{~nm}$, and fluorescence images were collected after cell autofluorescence was subtracted. The $m$ THPC fluorescence imaging of cells demonstrated that the $m$ THPC was internalized into cells. This finding means that the hydrophobic molecules were precipitating in aqueous environment and would be captured by any cells or tissues after administration. However, for $m$ THPCcontaining nanoparticles, no obvious intracellular $m$ THPC fluorescence was observed under the same conditions. These results show that the $m$ THPC-encapsulating nanoparticles can effectively alter $m$ THPC behaviors in aqueous environment.

The effectiveness of free and nanoencapsulated $m$ THPC in killing cultured rat C6 glioma cells was also evaluated. Exposure to 650 -nm light alone, or in combination with blank nanoparticles, did not affect the viability of the cells. Nanoparticles loaded with $m$ THPC also did not kill cells when incubated in the dark. However, rat C6 glioma cells were efficiently killed by exposure to $650-\mathrm{nm}$ light, in the presence of either free or nanoparticle-encapsulated $m$ THPC (Figure 3). The results indicate that $m$ THPC-containing nanoparticles cause significant cellular damage in the presence of light, and even a low concentration of $m$ THPC within the nanospheres, in combination with 650 -nm light, results in significant damage to the cells. Encapsulation of $m$ THPC into hydrogel polymers reduces molecular aggregation in physiological solutions, thus enhancing solubility and the production of singlet oxygen.

In summary, a novel synthetic approach for the fabrication of ultrafine hydrogel nanoparticles is presented. By a nonaqueous microemulsion method, the hydrophobic $m$ THPC was successfully encapsulated into hydrophilic polyacrylamide nanoparticles. Both dye aggregation within the watersoluble nanoparticles and dye leaching are minimized. Some potential advantages are available for these ultrafine $m$ THPC-encapsulating nanoparticles. Their ultrasmall size should not only help the nanoparticles evade the reticuloendothelial system (RES) but the produced singlet oxygen should more efficiently diffuse out of the nanoparticles so as to kill living tumor cells. The ultrafine nanoparticles could be potentially removed from the body by renal clearance. This decreases the accumulation risk of drug administration. These ultrafine, $m$ THPC-encapsulating polyacrylamide nanoparti- a)

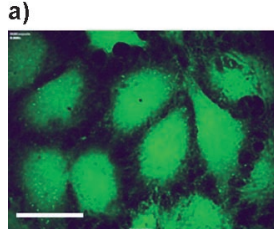

b)

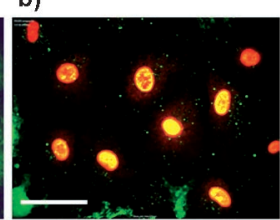

d)

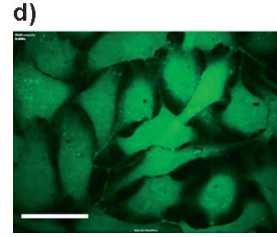

e)

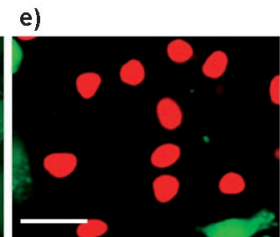

c)

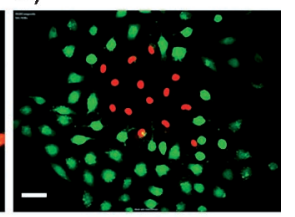

f)

Figure 3. In vitro cell controls and phototoxicity of $m$ THPC and its nanoparticles. a) Cells mixed with free $m$ THPC before light exposure; b) cells mixed with free $m$ THPC after light exposure; c) zoom out of (b); d) cells mixed with $m$ THPC NPs before light exposure; e) cells mixed with $m$ THPC NPs after light exposure. f) zoom out of (e). All the samples were exposed to $34-\mu \mathrm{W}$ laser illumination for $10 \mathrm{~min}$, and then images were taken every $2 \mathrm{~min}$ for up to $2 \mathrm{~h}$ to monitor cell death. The area of illumination under the $60 \times$ objective is around $160 \mu \mathrm{m} \times 160 \mu \mathrm{m}$. Scale bar for (a), (b), (d), and (e): $30 \mu \mathrm{m}$; scale bar for (c) and (f): $40 \mu \mathrm{m}$.

cles, in addition to their above-mentioned advantages, were as effective in killing cultured cancer cells as free $m$ THPC photosensitizer molecules. The above synthetic approaches could be easily expanded to encapsulate other hydrophobic drugs, with a variety of potential drug-delivery applications.

\section{Experimental Section}

All instruments, chemicals, and cell culture are detailed in the Supporting Information.

Blank polyacrylamide nanoparticles: AAm (0.8 g) and MBA $(0.09 \mathrm{~g})$ were directly emulsified into hexane $(40 \mathrm{~mL})$ containing AOT (11.25 g), and a clear solution was observed. The clear solution was stirred under argon for $20 \mathrm{~min}$. TMEDA $(20 \mu \mathrm{L})$ was added under argon. Then APS $\left(20 \mu \mathrm{L}, 100 \mathrm{mg} \mathrm{mL}^{-1}\right)$ was added to start polymerization. The reaction was allowed to proceed overnight. Once the polymerization reaction was completed, hexane was evaporated and the residue was resuspended in methanol. The suspension was washed with methanol $(4 \times 150 \mathrm{~mL})$. White solid was obtained. The size of blank nanoparticles was measured by using dynamic light scattering, which showed a mean size of $1 \mathrm{~nm}$ in diameter. TEM results indicated that these particles have $2 \mathrm{~nm}$ average diameter.

$m$ THPC-encapsulating nanoparticles: AAm (1.05 g) and MBA $(0.1 \mathrm{~g})$ were sonicated in methanol $(1.5 \mathrm{~mL})$. Then $m$ THPC $(6 \mathrm{mg})$ was dissolved in the above solution. AOT (11.118 g) was dissolved in hexane $(60 \mathrm{~mL})$. The methanol solution containing AAm, MBA, and $m$ THPC was then emulsified into the hexane solution. A clear brown solution was observed. Methanol and hexane were evaporated at room temperature. Then hexane $(60 \mathrm{~mL})$ was added to the residue to obtain a clear solution. The clear dispersion solution was stirred under argon for $20 \mathrm{~min}$. TMEDA $(20 \mu \mathrm{L})$ was added under argon. Then APS $\left(20 \mu \mathrm{L}, 100 \mu \mathrm{g} \mathrm{mL}^{-1}\right)$ was added to start polymerization. The reaction was allowed to proceed overnight. Once the polymerization reaction was completed, hexane was evaporated and the residue was resuspended in methanol. The suspension was washed with methanol $(4 \times 150 \mathrm{~mL})$, and a green solid was obtained.

In vitro PDT studies: The rat C6 glioma cells on glass cover slips were removed from the culture medium, rinsed carefully with Hanks $1 \times$ balanced salt solution and placed in the microscope 
chamber at $37^{\circ} \mathrm{C}$. The cells were covered with Hanks $1 \times$ balanced salt solution $(2 \mathrm{~mL})$ containing $10 \mathrm{~mm}$ pH buffer HEPES (4-(2hydroxyethyl)piperazine-1-ethanesulfonic acid), $0.1 \mu \mathrm{M}$ calcein AM, $10 \mu \mathrm{M}$ propidium iodide (PI), and $0.12 \mu \mathrm{M} m$ THPC or its NP. Calcein AM, a cell-permeant dye, was used to determine cell viability both before and after NP treatment. In live cells the nonfluorescent calcein AM is converted to a green-fluorescent calcein after acetoxymethyl ester hydrolysis by intracellular esterases. Meanwhile, PI marks the nuclei once the integrity of the cell membrane is compromised, that is, detecting dead or dying cells. Pre-exposure confocal microscope images were taken with an oil immersion $60 \times$ objective lens in two channels (488-nm excitation of the calcein converted from calcein AM, 568-nm excitation of the PI). The cells were exposed for $10 \mathrm{~min}$ at $650 \mathrm{~nm}$ with a power intensity of $34 \mu \mathrm{W}$. Afterwards, images were taken every $2 \mathrm{~min}$ for up to $2 \mathrm{~h}$ to monitor cell death. Finally, a $20 \times$ objective was used to broaden the field of view and allow concurrent comparison of the cells exposed to light along with those that had not been exposed.

Received: September 25, 2006

Published online: February 20, 2007

Keywords: antitumor agents · drug delivery - hydrogels . nanostructures $\cdot$ photodynamic therapy

[1] S. M. Moghmi, A. C. Hunter, J. C. Murray, Pharmacol. Rev. 2001, $53,283-318$.

[2] N. A. Peppas, J. Z. Hilt, A. Khademhosseini, R. Langer, $A d v$. Mater. 2006, 18, 1345-1360; I. Brigger, C. Dubernet, P. Couvreur, Adv. Drug Delivery Rev. 2002, 46, 631-651.
[3] W. M. Sharman, J. E. van Lier, C. M. Allen, Adv. Drug Delivery Rev. 2004, 56, 53-76; S. Gross, A. Gilead, A. Scherz, M. Meeman, Y. Salomon, Nat. Med. 2003, 9, 1327-1331; M. Monga, E. A. Sausville, Leukemia 2002, 16, 520-526; I. J. Macdonald, T. J. Dougherty, J. Porphyrins Phthalocyanines 2001, 5, 105-129; F. S. De Rosa, M. V. L. B. Bentley, Pharm. Res. 2000, 17, 1447-1455; W. M. Sharman, C. M. Allen, J. E. van Lier, Drug Discovery Today 1999, 4, 507-517; T. J. Dougherty, C. J. Gomer, B. W. Henderson, G. Jori, D. Kessel, M. Korbelik, J. Moan, Q. Peng, J. Natl. Cancer Inst. 1998, 90, 889-905.

[4] D. E. J. G. J. Dolmans, D. Fukumura, R. K. Jain, Nat. Rev. Cancer 2003, 3, 380-387.

[5] S. Hettiaratchy, J. Clarke, J. Taubeland, C. Besa, Br. Med. J. 2000, $320,1245$.

[6] Y. N. Konan, R. Cerny, J. Favet, M. Berton, R. Gurny, E. Allemann, Eur. J. Pharm. Biopharm. 2003, 55, 115-124; Y. N. Konan, M. Berton, R. Gurny, E. Allemann, Eur. J. Pharm. Sci. 2003,18, 241-249.

[7] M. P. Pileni, Nat. Mater. 2003, 2, 145-150; M. P. Pileni, Supramol. Sci. 1998, 5, 321-329; M. P. Pileni, Langmuir 1997, 13, 32663276; A. Hammouda, T. Gulik, M. P. Pileni, Langmuir 1995, 11, $3656-3659$.

[8] M. J. Moreno, E. Monson, R. G. Ramachandra, A. Rehemtulla, B. D. Ross, M. Philbert, R. J. Schneider, R. Kopelman, Sens. Actuators B 2003, 90, 82-89.

[9] J. Y. Chen, N. K. Mak, C. M. N. Yow, M. C. Fung, L. C. Chiu, W. N. Leung, N. H. Cheung, Photochem. Photobiol. 2000, 72, 541 547. 